\title{
Integration and Extension and Division
}

\author{
Space Creation for TV Programs
}

\author{
Ying Guo \\ Journalism and Information Communication School \\ Huazhong University of Science and Technology \\ Wuhan, China
}

\begin{abstract}
For a long time, more attentions have been paid to TV's art of time but not to the art of space. Actually TV technologies that become mature increasingly have not only achieved the span of time dimension but also created different spaces. This article takes the development course of TV programs as a timeline and the spaces built in the programs as research objects, analyzing diverse spaces formed in TV programs, namely, on-stage and off-stage spaces that are fused gradually, extension spaces brought by technical progress, backstage spaces meeting audiences' curiosity and independent spaces with suspense set. Going with technical development and changes of audiences, space elements that are rich in creativity have attracted eyeballs of TV programs gradually, and the TV programs have achieved its conversion from one single to multiple in order to create spaces. The multiple spaces have not only enriched contents of TV programs but also provided audients with multiple experience, which form an important part of the program and play a significant role in programs of narrations.
\end{abstract}

Keywords—TV program space; space creativity; TV narration

\section{INTRODUCTION}

Just like what Marx said, kill time by time. Since the birth of TV medium, millions and millions of audiences have been attracted by it relying on an extremely rapid speed. TV medium takes rapid time to kill the distance in spaces, and it has not only increased the transmission speed but also expanded the transmission scope. Especially with the emerging and spread of TV live technique, the boundary in space has been completely broken through, and different spaces are connected at the same time. For a long time, more attentions have been paid to TV's art of time but not to the art of space. Actually TV technologies that become mature increasingly have not only achieved the span of time dimension but also created different spaces. This article takes the development course TV programs as timeline for analysis, showing the evolution process and development trend of spaces for TV programs.

\section{INTERACTION AMONG AUDIENCES: BREAK THROUGH THE UNCHANGED SPACES ON STAGE AND OFF STAGE}

TV program builds spaces inside and outside it for audiences, when audiences watch programs before television, the television is another space for audiences. Through TV images, audiences are brought to the space that the program shows, a news scene, or face to face performances of players or anywhere around the world with the guide of TV lens.
Traveling from real space to a virtual one, TV becomes a bridge for connection.

In early days, there were no audiences for stages of TV programs, with the development of TV programs, audiences are invited to the program recording scene. Within the TV programs where only hosts or players can be seen, an important group has appeared: audience. For example, from variety shows such as Super Variety Show to talk program Tell What it Should be and puzzle programs as Quiz Show, all took audiences as an important element to the program. The participation of audiences is considered an important step to stress interaction with audiences and improve the on-the-spot effect.

Within TV programs in the stages, through actors and audiences are arranged in the same space - studio, yet the high stages and audients off stages have formed invisibly two psychological spaces, that is, performance space for actors on stages and watching space for audiences off stages. Just like what stated in Brecht's Alienation Effect theory, "It needs to purposely make a distance or barrier between actors and operas concerned, characters, audiences and operas shown so that actors and audiences can be separated from the pure psychedelic scenes, emotional experience or resonance, and act as onlookers to review characters and events shown and think and judge wisely so as to get deeper understanding of the society and life."1

For a long time, audiences on the spot hold the concept of "outsiders" to watch TV programs, whose experience is basically the same as that of audiences before televisions. Actors on stages and audiences off stages are divided into two spaces by a wall invisible, which are independent of each other, in lack of interaction or exchange. Sometimes, actors step down from stages to interact with audiences or invite audiences to stages for communications, where the space barriers are broken temporarily. However, once the interaction ends, two independence spaces where actors perform on stages and audiences off stages watch occur once again.

In the late period of the on-stage and off-stage unchanged spaces, the thought of interaction with audiences started to sprout, and TV programs teams gradually came to know the importance of audiences to improve the program effect, thus audiences gradually become an important element for the programs. For example, Happy Camp often invite audiences off stages to participate in programs on stages, Quiz Show set 
special section to ask for help from audiences, Tell What it Should be make audiences' questions be the most excellent part in the program. Till now, the stable "on-stage and off-stage" space has been broke through, and "on-stage and off-stage" spaces are still the basic elements for TV programs, whether the reality show, talk or emotion programs, the off-stage spaces where audiences stay have been playing more and more important role in the programs. The fusion of on-stage and offstage spaces is achieved through interaction between audiences and more program experience, and the "on-stage and off-stage" spaces will not be kept unchanged, who have become a state flowing and integral, in the meantime, the spaces change also promotes the changes of audiences' status, experience of interaction, activate the program atmosphere in scene and improve the program effect to show.

\section{VIDEO Play DEVICES: CREATE SPACES FOR EXTENSION}

The development of TV technologies and rich stage devices have expanded the creativity of TV program spaces, video play devices used in studio create another important space in addition to stages and audiences, which improves the single watching experience of audients brought by single studio for a long time. Technical progress has promoted the wide use of video play devices in TV programs, and large LED screens are often used to watch VCR in TV programs, the videos that have been shot will be played through large screen in programs, which keeps an effective integration with the contents in situ, creating a space outside stages. Through large screens, audiences off stages may talk or communicate with those in videos, and the scene has been moved to another space outside the studio, that is, it has broken through the limit of physical spaces, getting a brand new experience. LED screens that used in TV programs break through traditional program space where only stages can be provided, for audiences, there is an additional new space different from the space in situ to experience. Besides, seen from the narration of the programs, the LED screen used has become a tool to enrich the contents, which plays a unique role in improving stage effect and intensifying program atmosphere.

Early TV programs generally install a LED screen on the wall facing audiences on stages so as to guarantee them to watch the video. With technical development and progress of stage design, the LED position and sizes both become a part carefully designed by the program groups. As for the narration function, it has become more and more important though at the beginning it is taken as additional content and connection. For example, in the talk program Voice, LED screen is set to $360 \mathrm{o}$ round, stretching around, besides, the stage is divided into three spaces namely host, lecturer and youth representative. Here the LED screen, in addition to video play and stage function, is used to divide spaces. With LED screens used technically to divide the stage space, the position of host is weakened in the traditional stage space, and a round stage will provided to radiate audiences off stages, which break through the space barrier between stages and audiences off stages, bringing strong senses of experience and participation to audiences, and the interactive atmosphere in the programs is more than hot.

\section{PROPELLED BY CURIOSITY: DEVELOPMENT OF BACKSTAGE SPACE}

For a long time, studio backstage is mysterious and strange for audiences, what audiences can see are mostly actors on stages, yet the backstage such as what the backstage looks like and how actors rehearse are little disclosed in TV programs. In early programs, some measures were ever taken to expose the backstage to audiences, yet it is just a small part for the whole programs or used as tidbits. The real time to take the backstage as an independent space to build and participate in the narration of the programs started with the program I'm a Singer on Hunan Satellite TV Station. In the program, in addition to traditional on-stage and off-stage spaces, another space namely the backstage is created where celebrities make reparations and watch programs. For audiences, how celebrities make preparations and communicate have not been secrets, in such space, the narration generally will place a foundation for the coming contents, which will be suspense or express emotions to improve the program effect. In a word, what the backstage do are mostly to serve the performance on the stage, the better the backstage narration is, the better the performance effect on the stage is. Within the space where celebrities watch programs, their status are changed to what the same as audiences before $\mathrm{TV}$, and the audiences enjoy additional senses of accompany to spend the time together while watching programs. The transformation of stages from sacred fixed form to that of daily life not only makes audiences enjoy the carnival on stages through space shift, but also returns to natural calmness in normal life.

Since the program I'm a Singer, to create and make full use of backstage for narration has become a method widely adopted in many programs especially reality shows. In the programs, a lot of scenes at the backstage, which were thought secrete in early period, are adopted to show audiences directly, and rich contents are provided and the backstage space is taken as an important element for narration. The exposure of backstage spaces meet audiences' curiosity and thirst of peeping while creating new program contents, such watching experience is extremely important for reality show programs to develop.

\section{Suspense Setting: CREATE AN INDEPENDENT SPACE}

If the exposure of backstage is to better lower the distance between audiences and actors from vision to mentality, the creation of an independent space is to purposely separate audiences from actors. To set a relatively closed space looks like going against the exposure of TV program spaces and gap removal, actually it is a suspense setting in order to attract audiences. For example, the emotional program Happy Cube on Dongfang Satellite TV Station sets a vertical and transparent glass room in the middle of the stage, host and parties concerned stay in the glass room, mental-health counselors and others concerned stay at four corners of from outside the glass room. The TV program like this, how to remove mental worries of the parties concerned and make them reveal what they have in heart have been an important problem that is related to the program effect. The glass room setting creates a relatively closed space for the parties concerned, bringing them a sense of safety, on one hand, the 
accompanying of host can comfort the parties concerned, on the other hand, the host will feel easy to guide the parties concerned to achieve his or her work. As a relatively independent space, the glass room is placed on the stage, and the closed door hints fates of the parties concerned, whether the door will be opened or not has become a suspense what every audience holds in heart. In the meantime, the space structure-glass room also works to show tenets of the program, namely seek the way to brand new happiness through wise cube.

Beijing Satellite TV Station launches a large celebrity cross border music reality show program Cross-border King Singer, which also borrowed independent spaces to set suspense. Differing from the independent space built by Happy Cube on the stage, the Cross-border King Singer builds a space off stage mysterious for audiences, a two-storey space structure, which is a new progress whether in originality or technology. The first storey space for audition plays a significant role for the program narration: first, for a celebrity, it has a decisive right for him or her to access the high level stage successfully. A relatively closed space and toe-to-toe three judges create a relatively nervous atmosphere for the celebrity to audition, facing such a situation, audiences are as nervous as the celebrity. Yet the audiences who are parallel with the stage space know nothing about what has happened at the low-level space. How the celebrities that audiences like and support perform at the low level audition space and whether they will be elevated to the high level stage to perform or not can be fond only when the stage is elevated. The low level audition space becomes a key step for the program narration, which gather audiences on stages and before TV together, how the competition goes and what the fates of celebrities are, all of the suspense will be shown with surprises, the program reaches peaks one after another. Seen from the above, as an important element, the independent space is used in the program and accompanied with the program development and separated from stages or public spaces of audiences, which creates suspense in the relatively closed environment and serve the whole program.

\section{CONCLUSION}

Space is a basic element for TV programs. Initially there was only one space namely studio for TV programs, going with the technical development and the increased requirements of audiences to watch, TV programs gradually come to know space, an element with great creativity.

To invite audiences to record programs in studios create a space for audiences off stages. No doubt that the audiences watching programs off stages further enrich the TV images. The participation of audiences in the programs breaks through the on-stage and off-stage fixed spaces through interaction. Since then, two spaces which were stable ever begin to fuse, in following programs, to change the positions of traditional audiences' seats, low stage's height or distribute audiences' seats at different areas around the stage all can be regarded as efforts to break through the on-stage and off-stage spaces where there are communications. The fusion of on-stage and off-stage spaces has dispelled the mentality of outsiders of audiences who act as onlookers, enabling them better integrate to the contents and scope set for the programs.
Technical progress has promoted the expansion and extension of spaces. The application of LED screens show the charm of TV, and the structures outside studios further enrich the program contents and audiences' experience in scene. Though sitting in the studios or before TV, audiences may access another stages through LED screens, compared to the pure space fusion, the indefinite extension of spaces bring much better effect for the programs. Similarly, as the extension of the stages, the backstage exposes secrets that should be hidden to audiences, making them work in narration and meeting audiences' curiosity and thirst of peeping.

Spaces can be fused or expanded and created as well. According to the column type and needs of programs, to create a new space in the studio is another active attempt for the art of TV space. Space creation goes against space fusion, and the latter aims to eliminate the distance in space and mentality between on stage and off stage, actors and actors, the former aims to create distances through creating new relatively closed spaces so as to set suspense. Actually the creation of new spaces looks to separate audients from the program for a certain period, yet its ultimate goal is to attract continuous eyeballs of audiences through tantalizing them, leading the program effect to the highest point.

As for whether to break through the distance between spaces or to create new spaces, the space has become an integral part of the TV programs. During the program design, the space is considered as an important element, whose functions and roles become more and more important. The space effect will be finally shown in audiences' visual experience, participating in the narration of program invisibly, enriching the program contents and improving the program effect.

\section{REFERENCES}

[1] Zhu Liyuan, Contemporary Western Theories of Literature and Art, East China University Press, 2005, Enlarged Edition, pp202.

[2] Tan Tian, Tan Qing, TV Programs as Space Products [J] Modern Communication (Journal of Communication University of China, 2016,02 .

[3] Yan An, Discussion on Objective of TV Studies [J]. Modern Communication (Journal of Communication University of China, 2014,05.

[4] Nie Jiangwen, Space Division: Discussion on Reform of TV Talk Programs [J]. Media, 2014,07.

[5] Liu Lu, Studies on Space Elements of TV Programs [D]. Shandong University of Arts, 2014. 